\title{
Bulk detection of time-dependent topological transitions in quenched chiral models
}

\author{
Alessio D’Errico $\odot,{ }^{1}$ Francesco Di Colandrea $\odot,{ }^{1}$ Raouf Barboza $\odot,{ }^{1}$ Alexandre Dauphin $\odot,{ }^{2,},{ }^{*}$ Maciej Lewenstein $\odot{ }^{2,3}$ \\ Pietro Massignan $\odot{ }^{2,4, \dagger}{ }^{2}$ Lorenzo Marrucci $\odot{ }^{1,5}$ and Filippo Cardano ${ }^{1, \ddagger}$ \\ ${ }^{1}$ Dipartimento di Fisica, Università di Napoli Federico II, Complesso Universitario di Monte Sant’Angelo, Via Cintia, 80126 Napoli, Italy \\ ${ }^{2}$ ICFO (Institut de Ciencies Fotoniques), Barcelona Institute of Science and Technology, 08860 Castelldefels (Barcelona), Spain \\ ${ }^{3}$ ICREA (Institució Catalana de Recerca i Estudis Avançats), Passeig Lluis Companys 23, 08010 Barcelona, Spain \\ ${ }^{4}$ Departament de Física, Universitat Politècnica de Catalunya, Campus Nord B4-B5, 08034 Barcelona, Spain \\ ${ }^{5}$ CNR-ISASI, Institute of Applied Science and Intelligent Systems, Via Campi Flegrei 34, 80078 Pozzuoli (NA), Italy
}

(Received 29 January 2020; accepted 23 March 2020; published 4 May 2020)

\begin{abstract}
The topology of one-dimensional chiral systems is captured by the winding number of the Hamiltonian eigenstates. Here we show that this invariant can be read out by measuring the mean chiral displacement of a single-particle wave function that is connected to a fully localized one via a unitary and translation-invariant map. Remarkably, this implies that the mean chiral displacement can detect the winding number even when the underlying Hamiltonian is quenched between different topological phases. We confirm experimentally these results in a quantum walk of structured light.
\end{abstract}

DOI: 10.1103/PhysRevResearch.2.023119

\section{INTRODUCTION}

Topological systems are characterized by quantized and global features, known as topological invariants, which are robust to smooth perturbations [1]. Nonzero values of these invariants underlie a variety of remarkable physical phenomena [2], such as the quantization of transport properties in quantum Hall systems [3-5] or the appearance of robust edge states [6-8]. Promising applications in metrology, spintronics, and quantum computation [9-13] fueled intense research in materials exhibiting topological order [14], leading eventually to the development of artificial topological systems in a variety of physical architectures (e.g., cold atom [15], photonic $[16,17]$, mechanical [18], and polariton [19] systems).

Topological phases can be classified and related to a specific topological invariant in terms of the system symmetries and dimensionality $[20,21]$. The simulation and measurement of topological insulators in different quantum simulators is a very active field and different techniques allow one to characterize the topology, relying on the detection of edge states [22-25], center-of-mass anomalous displacements [26,27], vortex dynamics in reciprocal space [28], interferometry [29-31], surface scattering [32], time-of-flight measurements [33], and the mean chiral displacement [34,35].

The investigation of these exotic systems also benefited from the simulation of topological phases in simple and con-

\footnotetext{
*alexandre.dauphin@icfo.eu

†pietro.massignan@upc.edu

‡filippo.cardano2@unina.it
}

Published by the American Physical Society under the terms of the Creative Commons Attribution 4.0 International license. Further distribution of this work must maintain attribution to the author(s) and the published article's title, journal citation, and DOI. trollable quantum evolutions known as quantum walks (QWs) [36]. Interestingly, quantum walks can be engineered so as to host all topological phases of noninteracting systems in one dimension (1D) and two dimensions (2D) [37], providing also one of the simplest examples of periodically driven (Floquet) systems [38-40]. Their study led to a number of theoretical and experimental findings in the context of static and Floquet topological physics, such as the discovery of Floquet anomalous regime $[22,25,34,41]$.

In this article, we focus on 1D systems with chiral symmetry, whose topological invariant is the winding number $[42,43]$. Some of us showed in Refs. [34,35] that such invariant can be accurately detected by measuring the mean chiral displacement (MCD) of a single particle, provided that at a given time this is fully localized on a single lattice site. This method does not require any band filling or the application of an external force, and as such it is extremely versatile and has already found application in several experimental scenarios [44-50]. Here we show that this scheme works also for a much larger class of input states, which may be delocalized over many lattice sites. We indeed demonstrate that the input state can be any state that can be mapped to a localized wave function via a unitary and translation-invariant operator. Interestingly, such operator can also correspond to the evolution operator associated with an Hamiltonian different from the instantaneous one. As a consequence, we show that in a sudden dynamical transition between two chiral topological phases, hereafter referred to as quench, the mean chiral displacement signals the phase transition and, in the long time limit, eventually tracks the value of the winding number of the postquench Hamiltonian. After deriving these results, which apply to generic 1D chiral systems, we present an experiment validating our theory in a photonic quantum walk that implements a Floquet chiral model. 


\section{ONE-DIMENSIONAL CHIRAL SYSTEMS}

We consider a system possessing an external discrete degree of freedom, for example, its position along a 1D lattice, and an internal degree of freedom, representing the internal state within a given unit cell. These are associated respectively with Hilbert spaces $\mathcal{H}_{e}$, spanned by the states $|m\rangle(m \in \mathbb{Z})$, and $\mathcal{H}_{i}$, spanned by $\mathcal{D}$-dimensional vectors, where $\mathcal{D}$ is the internal dimension. We assume translational invariance, so that the Hamiltonian can be written as $H=\int \frac{d q}{2 \pi}|q\rangle\langle q| \mathcal{H}(q)$, where $q$ is the quasimomentum defined in the Brillouin zone (BZ) $[-\pi, \pi[$. The Hamiltonian exhibits chiral symmetry if there exists an operator $\Gamma$ which is local, squares to unity, and anticommutes with $H$ (i.e., it acts only on $\mathcal{H}_{i}$, and it satisfies $\Gamma^{2}=1$ and $\Gamma H=-H \Gamma$ ). A prototypical example of a chiral Hamiltonian is the Su-Schrieffer-Heeger model [51], describing the electron effective dynamics in a polyacetylene chain.

One-dimensional chiral systems can be characterized by a topological invariant, called the winding number, that in the reciprocal space reads

$$
v=\operatorname{Tr}\left[\Gamma \mathcal{H}^{-1} \partial_{q} \mathcal{H}\right]
$$

and may take arbitrary (positive or negative) integer values. Here, the trace runs over both the 1D external degree of freedom (BZ) and the $\mathcal{D}$-dimensional internal space.

\section{MEAN CHIRAL DISPLACEMENT AFTER A QUENCH}

As first demonstrated in Refs. [34,35], the winding of an arbitrary noninteracting $1 \mathrm{D}$ chiral model may be conveniently captured by a measurement of the mean chiral displacement,

$$
\mathcal{C}(t)=\sum_{j}\left\langle\psi_{j}(t)|\Gamma X| \psi_{j}(t)\right\rangle,
$$

where $X$ is the position operator (acting as $X|m\rangle=m|m\rangle$ ), and $\left\{\left|\psi_{j}(t)\right\rangle\right\}(j=1, \ldots, \mathcal{D})$ is a set of states such that $\left|\psi_{j}(t=0)\right\rangle=|0\rangle \otimes\left|\phi_{j}\right\rangle$, i.e., which at time $t=0$ are completely localized on the central unit cell of the lattice, and whose internal states $\phi_{j}$ form a complete basis of the unit cell [52]. In the long time limit, one finds $\mathcal{C}(t \rightarrow \infty) \rightarrow v$.

Here we generalize these findings by showing that the final result is independent from the early dynamics, provided that this is translation invariant. In particular, this covers the possibility of studying sudden quenches during the evolution. To be specific, let us consider that between time 0 and time $t_{c}>0$ the evolution is governed by a translation-invariant Hamiltonian $H_{1}$. We stress here that $H_{1}$ does not need to be chiral. At time $t_{c}$, a sudden quench is applied to the system, and at later times the evolution is governed by the chiral Hamiltonian $H_{2}$. An initially localized state $\left|\psi_{j}(t=0)\right\rangle=$ $|0\rangle \otimes\left|\phi_{j}\right\rangle$ will evolve as

$$
\left|\psi_{j}(t)\right\rangle=V_{2} V_{1}\left|\psi_{j}(t=0)\right\rangle=\int \frac{d q}{2 \pi} \mathcal{V}_{2}(q) \mathcal{V}_{1}(q)\left|\phi_{j}\right\rangle,
$$

where we have introduced the evolution operators $V_{2}=\int \frac{d q}{2 \pi}|q\rangle\langle q| \mathcal{V}_{2}(q)=e^{-i\left(t-t_{c}\right) \theta\left(t-t_{c}\right) H_{2}}$, and $V_{1}=\int \frac{d q}{2 \pi}|q\rangle$ $\langle q| \mathcal{V}_{1}(q)=e^{-i t \theta(t) \theta\left(t_{c}-t\right) H_{1}}$, with $\theta(t)$ the Heaviside function and $\hbar=1$. Since the position operator $X$ acts as $i \partial_{q}$ in Fourier space, the MCD at time $t$ is

$$
\mathcal{C}=\sum_{j} \int \frac{d q}{2 \pi}\left\langle\phi_{j}\left|\mathcal{V}_{1}^{\dagger} \mathcal{V}_{2}^{\dagger} \Gamma i \partial_{q}\left(\mathcal{V}_{2} \mathcal{V}_{1}\right)\right| \phi_{j}\right\rangle
$$

To proceed, we use $\partial_{q}\left(\mathcal{V}_{2} \mathcal{V}_{1}\right)=\left(\partial_{q} \mathcal{V}_{2}\right) \mathcal{V}_{1}+\mathcal{V}_{2}\left(\partial_{q} \mathcal{V}_{1}\right)$ and $V_{2}^{\dagger} \Gamma=\Gamma V_{2}$ (which holds for chiral Hamiltonians). Furthermore, since $\sum_{j}$ is effectively a trace over the internal space, we use the cyclic property of the trace and the fact that $\mathcal{V}_{1} \mathcal{V}_{1}^{\dagger}=\mathbb{1}$, and immediately obtain

$$
\mathcal{C}=\sum_{j} \int \frac{d q}{2 \pi}\left\langle\phi_{j}\left|\mathcal{V}_{2}^{\dagger} \Gamma\left(i \partial_{q} \mathcal{V}_{2}\right)+\mathcal{V}_{1}^{\dagger} \Gamma \mathcal{V}_{2}^{2}\left(i \partial_{q} \mathcal{V}_{1}\right)\right| \phi_{j}\right\rangle
$$

The first operator $\mathcal{V}_{2}^{\dagger} \Gamma\left(i \partial_{q} \mathcal{V}_{2}\right)$ is identical to the one discussed in Refs. [34,35], and in those works it was shown that it yields a contribution which converges asymptotically to the winding number. To proceed further, as we are mainly interested in the behavior of the MCD at long times, we will restrict ourselves to $t>t_{c}$, such that only $\mathcal{V}_{2}$ is time dependent. We notice that $\mathcal{V}_{2}^{2}=e^{-2 i\left(t-t_{c}\right) \theta\left(t-t_{c}\right) \mathcal{H}_{2}(q)}$, where $\mathcal{H}_{2}(q)$ is the quasimomentum space representation of $H_{2}$. The second operator in the integrand of Eq. (5) therefore generates contributions of the form $\int d q f(q) e^{i t g(q)}$, with $f$ and $g$ being smooth functions of $q$. These are rapidly oscillating terms with amplitude $\sim 1 / \sqrt{t}$ and zero mean, as guaranteed by the stationary phase formula [53]. In conclusion, we obtain

$$
\mathcal{C} \stackrel{t \gg t_{c}}{=} v+O\left(\frac{1}{\sqrt{t}}\right)
$$

where $v$ is the winding number of the chiral Hamiltonian $\mathrm{H}_{2}$ generating the dynamics after the quench. Equation (6) is the main finding of this paper. Although deceivingly simple, this has a series of remarkable consequences. A first one is the convergence of the MCD to the winding number of the Hamiltonian of an initially localized state on any cell of the lattice (as may be seen by taking $\mathcal{V}_{1}$ to be an instantaneous translation). A second one is the possibility of using the MCD as a topological marker even in the presence of multiple quenches during the evolution.

Let us now numerically illustrate our finding by two concrete examples of quenches, based on tight-binding models introduced in Ref. [35]. First, we consider the tight-binding model $\mathrm{SSH}_{4}$ sketched in Fig. 1(a), which has internal dimen$\operatorname{sion} \mathcal{D}=4$. For times $0<t<t_{c}$, we include in the Hamiltonian $H_{1}$ a staggering term, which explicitly breaks the chiral symmetry. The MCD signal therefore rapidly oscillates and settles around a nonquantized value. At time $t_{c}$, we remove the chiral-breaking term, and soon after the MCD signal is observed to converge to an integer value, which corresponds to the winding number of the model. In a second example, we analyze the SSH-LR sketched in Fig. 1(b). This is a variant of the SSH model which includes longer ranged tunnelings, and admits values of the winding number larger than 1 . Here we quench the Hamiltonian twice, and after each quench the MCD is shown to converge smoothly to the expected winding number within that phase.

Closely following the demonstration in Ref. [35] it can be further shown that, when $\mathcal{D}=2$, chiral symmetry ensures that the MCD is equal to twice the expectation value of the 
(a)
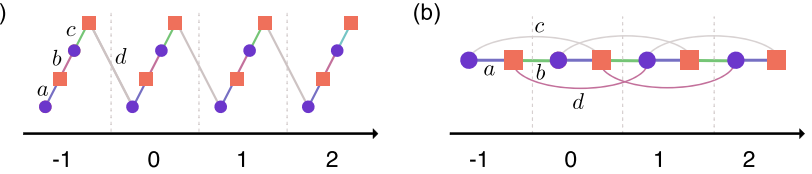

$\mathcal{C}$
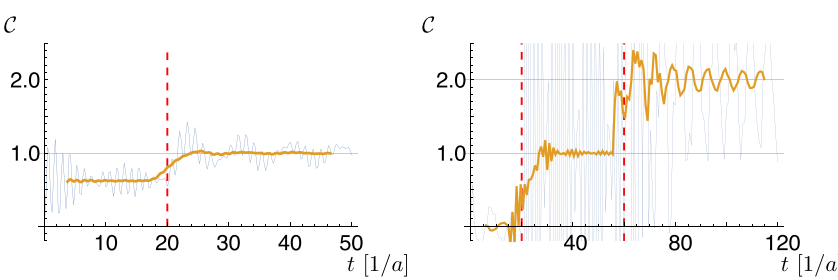

FIG. 1. MCD dynamics on simple models. (a) $\mathrm{SSH}_{4}$ model with chiral symmetry broken before $t_{c}=20 / a$, and restored after that [tunnelings: $a=b=c=\frac{d}{2}$, staggering: $\beta=a \theta\left(t_{c}-t\right)$ ]. (b) SSHLR model quenched from $v=0$ to 1 to 2 [tunnelings: $a=b, c=0$, and $d / a$ is quenched from 0.5 , to -0.5 , to 1.5 at times $t_{c, 1}=20 / a$ and $t_{c, 2}=60 / a$ ]. In both panels, the thick line is a moving average of the underlying data over a window of duration $10 / a$, which removes the fast oscillations.

operator $\Gamma X$ over a single arbitrary state localized within a single unit cell at $t=0$. Similarly for $\mathcal{D}>2$ the trace over the internal space may be replaced by twice the sum over the $\mathcal{D} / 2$ states from a single sublattice, provided the operator $V_{1}$ does not mix sublattices.

\section{QUENCHES IN A PHOTONIC QUANTUM WALK}

We verify our theoretical findings in a 1D chiral quantum walk, realized by engineering light propagation through a sequence of suitably patterned birefringent optical elements [27,54]. Specifically, as described in greater details in Ref. [27], we encode position states $|m\rangle$ (of the particle undergoing the QW) into Gaussian optical modes carrying a quantized amount of transverse wave vector $m \Delta k_{\perp}$. We keep the lattice spacing $\Delta k_{\perp} \ll k_{z}$, where $k_{z}$ is the longitudinal component of the wave vector, so that these modes remain confined along the $z$ axis. Coin states (that is the internal degree of freedom) instead are mapped into left and right circular polarizations, referred to as $\{|L\rangle,|R\rangle\}$. At each time step $t$ [55], the system evolution is determined by a combination of two transformations: a unitary operator $W$ performing a rotation of the polarization state only and a translation operator $T$ [54]. We implement $W$ by using a quarter wave plate (QWP) oriented with its fast axis parallel to the optical table. In the basis of circular polarizations, we have

$$
W=\frac{1}{\sqrt{2}}\left(\begin{array}{ll}
1 & i \\
i & 1
\end{array}\right) .
$$

The translation operator $T$ is implemented by liquid-crystal polarization gratings, referred to as $g$-plates [27], whose action is described by the operator

$$
T(\delta) \equiv\left(\begin{array}{cc}
\cos (\delta / 2) & i \sin (\delta / 2) \hat{t} \\
i \sin (\delta / 2) \hat{t}^{\dagger} & \cos (\delta / 2)
\end{array}\right),
$$

where $\hat{t} / \hat{t}^{\dagger}$ are, respectively, the (polarization-independent) left and right translation operators, acting as $\hat{t}|m\rangle=|m-1\rangle$
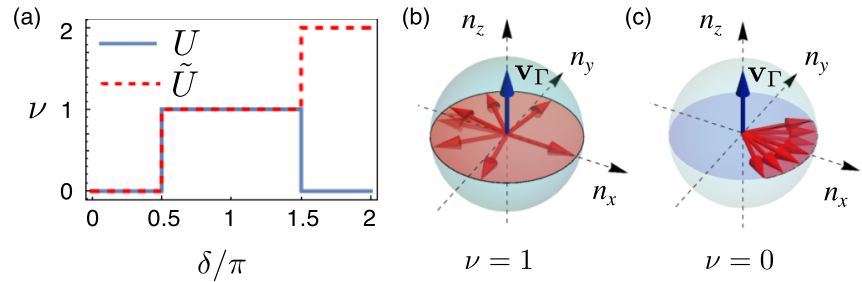

FIG. 2. Quantum walk winding numbers: (a) Dependence of the winding number on the parameter $\delta$ for protocols $U$ and $\tilde{U}$, as indicated in the legend. [(b), (c)] On the Poincaré sphere, we plot eigenstates $\mathbf{n}(q)$ of the evolution operator $U$, in two cases $\delta=\pi$ and $\delta=\pi / 4$, which have windings 1 and 0 , respectively. These states are positioned on a single plane, perpendicular to the vector $\mathbf{v}_{\Gamma}\left(\Gamma=\mathbf{v}_{\Gamma} \cdot \boldsymbol{\sigma}\right)$.

and $\hat{t}^{\dagger}|m\rangle=|m+1\rangle$, and $\delta$ is the plate optical retardation, which can be tuned by a voltage applied to the cell [56].

A quantum walk is defined in terms of the operator $U$ describing the evolution of a single time step. In our experiments, we consider the two protocols $U(\delta)=T(\delta) W$ and $\tilde{U}(\delta)=\sqrt{T(\delta)} W \sqrt{T(\delta)}$. Both feature chiral symmetry, with their winding number $v$ depending on $\delta$ as shown in the phase diagrams in Fig. 2(a) [34]. In Figs. 2(b) and 2(c), we plot the eigenstates $\mathbf{n}(q)$ of protocol $U$, in two illustrative cases with $\delta=\pi$ and $\delta=\pi / 4$. Vectors $\pm \mathbf{v}_{\Gamma}$ individuate the eigenstates of the chiral symmetry operator $\Gamma$ that we refer to as $|+\rangle$ and $|-\rangle$, respectively.

These QWs are simulated in the setup sketched in Fig. 3(a). A laser-light beam (wavelength $\lambda=632 \mathrm{~nm}$ ) with beam waist $\omega_{0} \simeq 5 \mathrm{~mm}$ propagates through a sequence of QWPs and $g$-plates, arranged so as to realize either protocol $U$ or $\tilde{U}$.

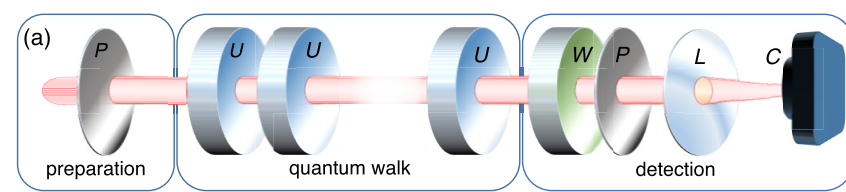

(b)
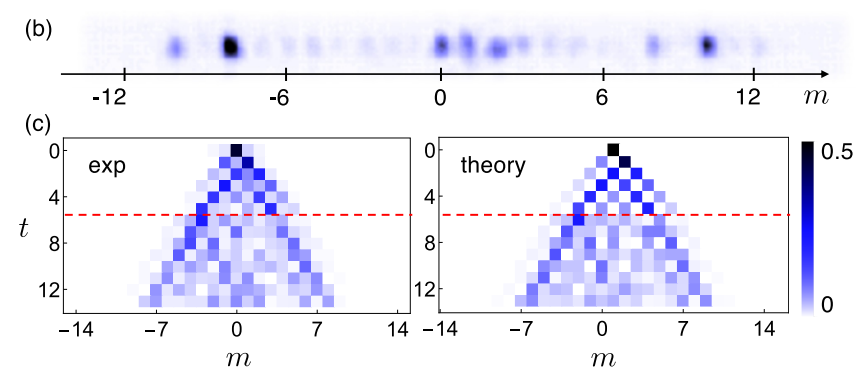

FIG. 3. Experimental setup and chiral probability distributions. (a) In our experiment, the input beam is prepared with a polarizer $(P)$, and then it performs a QW generated by either $U$ or $\tilde{U}$ (see main text). Finally, the chiral polarization components are analyzed with a QWP (W) and a polarizer, and the light intensity is recorded on a camera (C) placed in the focal plane of a lens (L). (b) Representative intensity distribution recorded by the camera. (c) Probability distributions $P_{-}(m)$ for a QW with a quench (at the fifth step; see the red dashed line) from the chiral protocol $U(\pi)$ to the chiral protocol $U(2 \pi / 5)$. Experimental results (left) are compared to numerical simulations (right). 
Before entering the QW setup, the optical field corresponds to the spatial mode $|m=0\rangle$, thus realizing the localized initial conditions given in the definition of the MCD [see Eq. (2)]. A polarizer guarantees that its polarization is horizontal. At the exit of the walk, a QWP and a polarizer, both mounted on rotating mounts, allow us to analyze individual polarization components associated with $|+\rangle$ and $|-\rangle$. After the polarization analysis stage, we place a lens and a camera to record light intensity in the focal plane. Here, the field intensity distribution features spatially separated Gaussian spots, each corresponding to a mode $|m\rangle$ [see Fig. 3(b)]. The overlap between the modes in the lens focal plane can be adjusted by tuning the spatial period of the gratings $\Lambda$. Choosing $\Lambda \simeq \omega_{0}=5 \mathrm{~mm}$ is sufficient to make this overlap negligible [27]. Recorded intensity patterns are processed to extract the particle probability distributions $P_{ \pm}(m)$ associated with chiral polarization states $| \pm\rangle$, respectively [see Fig. 3(c)]. We measure these distributions at each time step $t$, we compute the mean value of their difference, and we obtain the MCD defined in Eq. (2):

$$
\mathcal{C}(t)=\sum_{m} 2 m\left[P_{+}(m, t)-P_{-}(m, t)\right] .
$$

As discussed above, in our QW (having a unit cell with $\mathcal{D}=$ 2), the sum in Eq. (2) can be replaced by a single measurement, considering an arbitrary input state localized on a single unit cell at $t=0$. In our experiments, the polarization state of the light beam at the QW entrance is $(|L\rangle+|R\rangle) / \sqrt{2}$, that is a horizontal polarization. The first experimental validation of Eq. (6) is obtained by using the first five steps of our platform to implement the nonchiral protocol $T(3 \pi / 4) S$, where $S=\sigma_{x}$ is the operator associated with a half wave plate. The remaining part of the setup is arranged so as to perform eight steps of the QW $U(\pi)$. The results are shown in Fig. 4(a). We observe that after few time steps the MCD oscillates around $v$, with an amplitude getting smaller as $t$ increases.

Finally, we perform experiments to study quenches between different topological phases of both $U$ and $\tilde{U}$ protocols. In Figs. 4(b)-4(d), we show the evolution of the MCD in QWs where at the sixth step the value of $\delta$ has changed. In doing so, the system evolves under evolution operators associated with different winding numbers (see the figure caption for more details). All the experimental data correctly reproduce the oscillating behavior featured by theoretical simulations, showing that in a quench architecture the detection of the MCD allows one to monitor the winding number, faithfully signaling dynamical phase transitions. Error bars are the mean standard error (MSE) obtained from a set of four repeated measurements, each experiment being performed by realigning all plates in the QW setup. A few experimental points are more than three standard deviations away from values simulated numerically. This is ascribed to the presence of imperfections, such as defects in our plates, that induce deviations which are systematic and hence not taken into account in our experimental estimate of statistical uncertainties. The number of QW steps performed in this experiment was mainly limited by the number of liquid-crystal plates at our disposal, yet it was sufficient to provide a clean experimental demonstration of our main finding, contained in Eq. (6). In principle, one could reach a larger number of steps, provided (a)

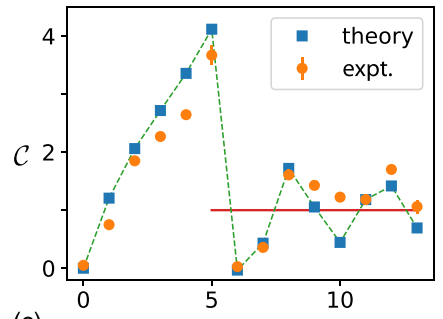

(c)

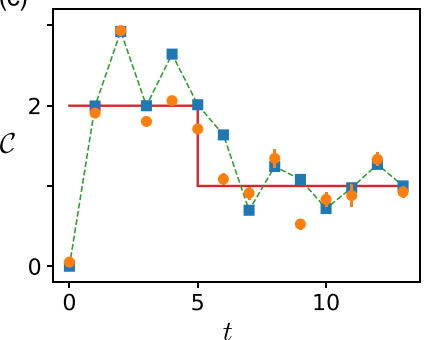

(b)

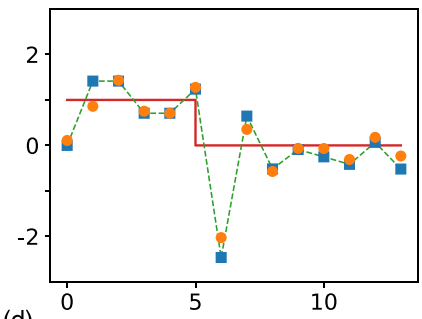

(d)

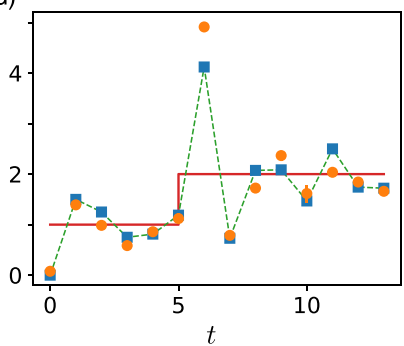

FIG. 4. MCD in quenched QWs. MCD in quantum walks governed by protocol $V_{1}$ from steps 1 to 5 and by protocol $V_{2}$ from steps 6 to 13. The winding number $v$ of each protocol is shown with red lines. (a) $V_{1}=T(3 \pi / 4) S$ is nonchiral (therefore its winding is not defined), $V_{2}=U(\pi)$. (b) $V_{1}=U(\pi), V_{2}=U(2 \pi / 5)$. (c) To study quenches between nontrivial phases, we chose here $V_{1}=\tilde{U}(7 \pi / 4)$ and $V_{2}=\tilde{U}(\pi)$, having winding numbers 2 and 1 , respectively. (d) Initial and final evolutions are swapped with respect to panel (c). In all plots, experimental data (orange dots) are compared with theoretical simulations (blue squares). In panels (c) and (d), an extra plate implementing the operator $T\left[\left(\delta_{2}-\delta_{1}\right) / 2\right]$ was inserted between the fifth and sixth steps.

that the condition $m \Delta k_{\perp} \ll k_{z}$ holds true for each mode $|m\rangle$. In our case, practical issues were the limited transmittance of the liquid-crystal plates, which can be improved with dedicated antireflection coatings, and the additional relative phase shifts accumulated by different modes during freespace propagation. These could be eliminated by introducing suitable imaging systems between consecutive steps. Further details can be found in Ref. [27].

\section{CONCLUSIONS AND OUTLOOKS}

We have shown that the mean chiral displacement is a powerful tool to probe the topology of chiral 1D systems whose initial state is connected to a localized one via a unitary and translation-invariant transformation. As such, the MCD can be used as a topological marker in experiments where the underlying Hamiltonian is suddenly quenched between different topological phases, like those studying topological systems out of equilibrium and dynamical topological phase transitions [57-59]. Remarkably, we showed that the MCD always performs damped oscillations around the winding number of the instantaneous Hamiltonian. In the future, it would be interesting to apply similar ideas to systems with higher dimensional internal states and to nonunitary processes [60-62].

\section{ACKNOWLEDGMENTS}

A.D'E., R.B., L.M., and F.C. acknowledge financial support from the European Union Horizon 2020 program, 
under European Research Council (ERC) Grant No. 694683 (PHOSPhOR). A.D. and M.L. acknowledge financial support from the Spanish Ministry MINECO (National Plan 15 FISICATEAMO Grant No. FIS2016-79508-P and SEVERO OCHOA Grant No. SEV-2015-0522, FPI), European Social Fund, Fundació Cellex, Generalitat de Catalunya (AGAUR Grant No. 2017 SGR 1341 and CERCA/Program),
ERC AdG NOQIA, and the National Science Centre, Poland-Symfonia Grant No. 2016/20/W/ST4/00314. A.D. is financed by a Juan de la Cierva fellowship (No. IJCI-2017-33180). P.M. acknowledges support by the "Ramón y Cajal" program and by the Spanish MINECO (Grant No. FIS2017-84114-C2-1-P). A.D., M.L., and P.M. acknowledge support from EU FEDER Quantumcat.
[1] M. Z. Hasan and C. L. Kane, Colloquium: Topological insulators, Rev. Mod. Phys. 82, 3045 (2010).

[2] X.-L. Qi and S.-C. Zhang, Topological insulators and superconductors, Rev. Mod. Phys. 83, 1057 (2011).

[3] K. von Klitzing, G. Dorda, and M. Pepper, New Method for High-Accuracy Determination of the Fine-Structure Constant Based on Quantized Hall Resistance, Phys. Rev. Lett. 45, 494 (1980).

[4] C. L. Kane and E. J. Mele, Quantum Spin Hall Effect in Graphene, Phys. Rev. Lett. 95, 226801 (2005).

[5] B. A. Bernevig, T. L. Hughes, and S.-C. Zhang, Quantum spin Hall effect and topological phase transition in $\mathrm{HgTe}$ quantum wells, Science 314, 1757 (2006).

[6] B. I. Halperin, Quantized Hall conductance, current-carrying edge states, and the existence of extended states in a twodimensional disordered potential, Phys. Rev. B 25, 2185 (1982).

[7] M. Hafezi, S. Mittal, J. Fan, A. Migdall, and J. M. Taylor, Imaging topological edge states in silicon photonics, Nat. Photon. 7, 1001 (2013).

[8] M. Mancini, G. Pagano, G. Cappellini, L. Livi, M. Rider, J. Catani, C. Sias, P. Zoller, M. Inguscio, M. Dalmonte, and L. Fallani, Observation of chiral edge states with neutral fermions in synthetic Hall ribbons, Science 349, 1510 (2015).

[9] K. von Klitzing, The quantized Hall effect, Rev. Mod. Phys. 58, 519 (1986).

[10] M. H. Freedman, A. Kitaev, and Z. Wang, Simulation of topological field theories by quantum computers, Commun. Math. Phys. 227, 587 (2002).

[11] C. Nayak, S. H. Simon, A. Stern, M. Freedman, and S. Das Sarma, Non-Abelian anyons and topological quantum computation, Rev. Mod. Phys. 80, 1083 (2008).

[12] A. Fert, Nobel lecture: Origin, development, and future of spintronics, Rev. Mod. Phys. 80, 1517 (2008).

[13] J. K. Pachos, Introduction to Topological Quantum Computation (Cambridge University Press, Cambridge, UK, 2012).

[14] M. G. Vergniory, L. Elcoro, C. Felser, N. Regnault, B. A. Bernevig, and Z. Wang, A complete catalogue of high-quality topological materials, Nature (London) 566, 480 (2019).

[15] N. R. Cooper, J. Dalibard, and I. B. Spielman, Topological bands for ultracold atoms, Rev. Mod. Phys. 91, 015005 (2019).

[16] M. C. Rechtsman, J. M. Zeuner, Y. Plotnik, Y. Lumer, D. Podolsky, F. Dreisow, S. Nolte, M. Segev, A. Szameit, Photonic Floquet topological insulators, Nature (London) 496, 196 (2013).

[17] T. Ozawa, H. M. Price, A. Amo, N. Goldman, M. Hafezi, L. Lu, M. C. Rechtsman, D. Schuster, J. Simon, O. Zilberberg, and I. Carusotto, Topological photonics, Rev. Mod. Phys. 91, 015006 (2019).
[18] R. Süsstrunk and S. D. Huber, Observation of phononic helical edge states in a mechanical topological insulator, Science 349, 47 (2015).

[19] P. St-Jean, V. Goblot, E. Galopin, A. Lemaitre, T. Ozawa, L. Le Gratiet, I. Sagnes, J. Bloch, and A. Amo, Lasing in topological edge states of a one-dimensional lattice, Nat. Photon. 11, 651 (2017).

[20] A. Kitaev, Periodic table for topological insulators and superconductors, in Advances in Theoretical Physics: Landau Memorial Conference, edited by V. Lebedev and M. Feigel'man, AIP Conf. Proc. No. 1134 (AIP, New York, 2009), p. 22.

[21] S. Ryu, A. P. Schnyder, A. Furusaki, and A. W. W. Ludwig, Topological insulators and superconductors: Tenfold way and dimensional hierarchy, New J. Phys. 12, 065010 (2010).

[22] T. Kitagawa, M. A. Broome, A. Fedrizzi, M. S. Rudner, E. Berg, I. Kassal, A. Aspuru-Guzik, E. Demler, and A. G. White, Observation of topologically protected bound states in photonic quantum walks, Nat. Commun. 3, 882 (2012).

[23] M. Leder, C. Grossert, L. Sitta, M. Genske, A. Rosch, and M. Weitz, Real-space imaging of a topologically protected edge state with ultracold atoms in an amplitude-chirped optical lattice, Nat. Commun. 7, 13112 (2016).

[24] E. J. Meier, F. A. An, and B. Gadway, Observation of the topological soliton state in the Su-Schrieffer-Heeger model, Nat. Commun. 7, 13986 (2016).

[25] L. Xiao, X. Zhan, Z. H. Bian, K. K. Wang, X. Zhang, X. P. Wang, J. Li, K. Mochizuki, D. Kim, N. Kawakami et al., Observation of topological edge states in parity-time-symmetric quantum walks, Nat. Phys. 13, 1117 (2017).

[26] M. Aidelsburger, M. Lohse, C. Schweizer, M. Atala, J. T. Barreiro, S. Nascimbène, N. R. Cooper, I. Bloch, and N. Goldman, Measuring the Chern number of Hofstadter bands with ultracold bosonic atoms, Nat. Phys. 11, 162 (2015).

[27] A. D'Errico, F. Cardano, M. Maffei, A. Dauphin, R. Barboza, C. Esposito, B. Piccirillo, M. Lewenstein, P. Massignan, and L. Marrucci, Two-dimensional topological quantum walks in the momentum space of structured light, Optica 7, 108 (2020).

[28] N. Fläschner, D. Vogel, M. Tarnowski, B. S. Rem, D.-S. Lühmann, M. Heyl, J. C. Budich, L. Mathey, K. Sengstock, C. Weitenberg et al., Observation of dynamical vortices after quenches in a system with topology, Nat. Phys. 14, 265 (2017).

[29] D. A. Abanin, T. Kitagawa, I. Bloch, and E. Demler, Interferometric Approach to Measuring Band Topology in 2D Optical Lattices, Phys. Rev. Lett. 110, 165304 (2013).

[30] M. Atala, M. Aidelsburger, J. T. Barreiro, D. A. Abanin, T. Kitagawa, E. Demler, and I. Bloch, Direct measurement of the Zak phase in topological Bloch bands, Nat. Phys. 9, 795 (2013).

[31] E. Flurin, V. V. Ramasesh, S. Hacohen-Gourgy, L. S. Martin, N. Y. Yao, and I. Siddiqi, Observing Topological Invariants 
Using Quantum Walks in Superconducting Circuits, Phys. Rev. X 7, 031023 (2017).

[32] S. Barkhofen, T. Nitsche, F. Elster, L. Lorz, A. Gábris, I. Jex, and C. Silberhorn, Measuring topological invariants in disordered discrete-time quantum walks, Phys. Rev. A 96, 033846 (2017).

[33] L. Wang, A. A. Soluyanov, and M. Troyer, Proposal for Direct Measurement of Topological Invariants in Optical Lattices, Phys. Rev. Lett. 110, 166802 (2013).

[34] F. Cardano, A. D’Errico, A. Dauphin, M. Maffei, B. Piccirillo, C. de Lisio, G. De Filippis, V. Cataudella, E. Santamato, L. Marrucci, M. Lewenstein, and P. Massignan, Detection of Zak phases and topological invariants in a chiral quantum walk of twisted photons, Nat. Commun. 8, 15516 (2017).

[35] M. Maffei, A. Dauphin, F. Cardano, M. Lewenstein, and P. Massignan, Topological characterization of chiral models through their long time dynamics, New J. Phys. 20, 013023 (2018).

[36] Y. Aharonov, L. Davidovich, and N. Zagury, Quantum random walks, Phys. Rev. A 48, 1687 (1993).

[37] T. Kitagawa, M. S. Rudner, E. Berg, and E. Demler, Exploring topological phases with quantum walks, Phys. Rev. A 82, 033429 (2010).

[38] T. Kitagawa, E. Berg, M. Rudner, and E. Demler, Topological characterization of periodically driven quantum systems, Phys. Rev. B 82, 235114 (2010).

[39] N. Goldman and J. Dalibard, Periodically Driven Quantum Systems: Effective Hamiltonians and Engineered Gauge Fields, Phys. Rev. X 4, 031027 (2014).

[40] A. Eckardt, Colloquium: Atomic quantum gases in periodically driven optical lattices, Rev. Mod. Phys. 89, 011004 (2017).

[41] F. Cardano, M. Maffei, F. Massa, B. Piccirillo, C. de Lisio, G. De Filippis, V. Cataudella, E. Santamato, and L. Marrucci, Dynamical moments reveal a topological quantum transition in a photonic quantum walk, Nat. Commun. 7, 11439 (2015).

[42] J. K. Asbóth, L. Oroszlány, and A. Pályi, A Short Course on Topological Insulators: Band Structure and Edge States in One and Two Dimensions (Springer, Berlin, 2016).

[43] J. K. Asbóth and H. Obuse, Bulk-boundary correspondence for chiral symmetric quantum walks, Phys. Rev. B 88, 121406(R) (2013).

[44] X. Wang, L. Xiao, X. Qiu, K. Wang, W. Yi, and P. Xue, Detecting topological invariants and revealing topological phase transitions in discrete-time photonic quantum walks, Phys. Rev. A 98, 013835 (2018).

[45] Y. Wang, Y.-H. Lu, F. Mei, J. Gao, Z.-M. Li, H. Tang, S.-L. Zhu, S. Jia, and X.-M. Jin, Direct Observation of Topology from Single-Photon Dynamics, Phys. Rev. Lett. 122, 193903 (2019).
[46] E. J. Meier, F. A. An, A. Dauphin, M. Maffei, P. Massignan, T. L. Hughes, and B. Gadway, Observation of the topological Anderson insulator in disordered atomic wires, Science 362, 929 (2018).

[47] D. Xie, W. Gou, T. Xiao, B. Gadway, and B. Yan, Topological characterizations of an extended Su-Schrieffer-Heeger model, npj Quantum Inf. 5, 55 (2019).

[48] L. Zhou and J. Pan, Non-Hermitian Floquet topological phases in the double-kicked rotor, Phys. Rev. A 100, 053608 (2019).

[49] R. W. Bomantara, L. Zhou, J. Pan, and J. Gong, Coupledwire construction of static and Floquet second-order topological insulators, Phys. Rev. B 99, 045441 (2019).

[50] D. Xie, T.-S. Deng, T. Xiao, W. Gou, T. Chen, W. Yi, and B. Yan, Topological Quantum Walks in Momentum Space with a Bose-Einstein Condensate, Phys. Rev. Lett. 124, 050502 (2020).

[51] W. P. Su, J. R. Schrieffer, and A. J. Heeger, Soliton excitations in polyacetylene, Phys. Rev. B 22, 2099 (1980).

[52] In the case $\mathcal{D}=2$, Eq. (2) yields twice the value obtained by using the definition provided in Ref. [34].

[53] R. Wong, Asymptotic Approximations of Integrals, Classics in Applied Mathematics (Society for Industrial and Applied Mathematics, New York, 2001).

[54] F. Cardano, F. Massa, H. Qassim, E. Karimi, S. Slussarenko, D. Paparo, C. de Lisio, F. Sciarrino, E. Santamato, R. W. Boyd, and L. Marrucci, Quantum walks and wave packet dynamics on a lattice with twisted photons, Sci. Adv. 1, e1500087 (2015).

[55] With a slight abuse of notation, we use the same variable $t$ for both the continuous time and discrete time steps.

[56] B. Piccirillo, V. D'Ambrosio, S. Slussarenko, L. Marrucci, and E. Santamato, Photon spin-to-orbital angular momentum conversion via an electrically tunable $q$-plate, Appl. Phys. Lett. 97, 241104 (2010).

[57] K. Wang, X. Qiu, L. Xiao, X. Zhan, Z. Bian, W. Yi, and P. Xue, Simulating Dynamic Quantum Phase Transitions in Photonic Quantum Walks, Phys. Rev. Lett. 122, 020501 (2019).

[58] M. Heyl, Dynamical quantum phase transitions: A brief survey, Europhys. Lett. 125, 26001 (2019).

[59] M. Tarnowski, F. N. Ünal, N. Fläschner, B. S. Rem, A. Eckardt, K. Sengstock, and C. Weitenberg, Measuring topology from dynamics by obtaining the Chern number from a linking number, Nat. Commun. 10, 1728 (2019).

[60] K. Yokomizo and S. Murakami, Non-Bloch Band Theory of Non-Hermitian Systems, Phys. Rev. Lett. 123, 066404 (2019).

[61] S. Longhi, Probing non-Hermitian skin effect and non-Bloch phase transitions, Phys. Rev. Research 1, 023013 (2019).

[62] Z. Gong, Y. Ashida, K. Kawabata, K. Takasan, S. Higashikawa, and M. Ueda, Topological phases of non-Hermitian systems, Phys. Rev. X 8, 031079 (2018). 